PROCEEDINGS OF THE

AMERICAN MATHEMATICAL SOCIETY

Volume 125, Number 9, September 1997, Pages 2809-2813

S 0002-9939(97)04031-8

\title{
SEMI-FREE ACTIONS OF ZERO-DIMENSIONAL COMPACT GROUPS ON MENGER COMPACTA
}

\author{
KATSURO SAKAI
}

(Communicated by James West)

\begin{abstract}
Let $\mu^{n}$ be the $n$-dimensional universal Menger compactum, $X$ a $Z$-set in $\mu^{n}$ and $G$ a metrizable zero-dimensional compact group with $e$ the unit. It is proved that there exists a semi-free $G$-action on $\mu^{n}$ such that $X$ is the fixed point set of every $g \in G \backslash\{e\}$. As a corollary, it follows that each compactum with $\operatorname{dim} \leqslant n$ can be embedded in $\mu^{n}$ as the fixed point set of some semi-free $G$-action on $\mu^{n}$.
\end{abstract}

In [Dr], Dranishnikov showed that every metrizable zero-dimensional compact group $G$ acts freely on the $n$-dimensional universal Menger compactum ${ }^{1} \mu^{n}$ (cf. [Sa]). Here we consider the fixed point sets of semi-free actions ${ }^{2}$ of $G$ on $\mu^{n}$. A closed set $X$ in $\mu^{n}$ is called a $Z$-set if there are maps $f: \mu^{n} \rightarrow \mu^{n} \backslash X$ arbitrarily close to id. The following is our result:

Theorem. Let $G$ be a metrizable zero-dimensional compact group with e the unit and $X$ a $Z$-set in $\mu^{n}$. Then there exists a semi-free $G$-action on $\mu^{n}$ such that $X$ is the fixed point set of every $g \in G \backslash\{e\}$.

By [Be, 2.3.8], each compactum $X$ with $\operatorname{dim} X \leqslant n$ can be embedded in $\mu^{n}$ as a $Z$-set. Then we have the following:

Corollary. Let $G$ be a metrizable zero-dimensional compact group. Each compactum $X$ with $\operatorname{dim} X \leqslant n$ can be embedded in $\mu^{n}$ as the fixed point set of some semi-free $G$-action on $\mu^{n}$.

In the proof below, for two simplicial complexes $K$ and $L, K \times L$ denotes the simplicial complex defined as the barycentric subdivision of the cell complex $\{\sigma \times \tau \mid$ $\sigma \in K, \tau \in L\}$. For any simplicial map $f: K \rightarrow L$, the simplicial mapping cylinder of $f$ is denoted by $M(f)$ (cf. [Wh, $\S 6]$ ). Notice that $K$ and $L$ are subcomplexes of $M(f)$. By $K^{(0)}$, we denote the set of vertices (0-skeleton) of $K$.

Proof of Theorem. We may only consider the case that $G$ is non-trivial, i.e., $G \neq$ $\{e\}$. By a well-known theorem of Pontryagin $[\mathrm{Po}, \S 46, \mathrm{C}], G$ is the inverse limit of

Received by the editors April 16, 1994 and, in revised form, April 28, 1996.

1991 Mathematics Subject Classification. Primary 54F15, 54H25, 54H15; Secondary 57S10, $22 \mathrm{C} 05$.

Key words and phrases. The fixed point set, semi-free action, 0-dimensional compact group, the $n$-dimensional universal Menger compactum.

${ }^{1} \mathrm{~A}$ compactum is a compact metrizable space.

${ }^{2}$ An action of $G$ on a space $X$ is called semi-free if the isotropy subgroup $G_{x}$ of $G$ at each $x \in X$ is trivial or all of $G$, where $G_{x}=\{g \in G \mid g x=x\}$. 
an inverse sequence of non-trivial finite groups

$$
G_{1} \stackrel{\varphi_{1}}{\longleftarrow} G_{2} \stackrel{\varphi_{2}}{\longleftarrow} G_{3} \stackrel{\varphi_{3}}{\longleftarrow} \cdots
$$

whence $G$ is a subgroup of $\prod_{i \in \mathbb{N}} G_{i}$. We denote the unit of $G_{i}$ by $e_{i}$. For each $i \in \mathbb{N}$, we denote

$$
G_{i}^{\prime}=\left\{\left(\varphi_{1} \cdots \varphi_{i-1}(g), \cdots, \varphi_{i-1}(g), g\right) \mid g \in G_{i}\right\} \subset G_{1} \times \cdots \times G_{i}
$$

and $e_{i}^{\prime}=\left(e_{1}, \cdots, e_{i}\right) \in G_{i}^{\prime} \subset G_{1} \times \cdots \times G_{i}$. Let $L_{i}$ be the $n$-dimensional $(n-1)$ connected simplicial free $G_{i}$-complex. Such a complex is defined in [Sa]. Then the $G_{i}$-action on $L_{i}$ extends naturally to the simplicial semi-free $G_{i}$-action on the cone $v_{i} * L_{i}$ over $L_{i}$ such that the cone vertex $v_{i}$ is the unique fixed point of every $g \in G_{i} \backslash\left\{e_{i}\right\}$. For each $i \in \mathbb{N}$, choose a vertex $u_{i}$ of $L_{i}$.

By Freudenthal's Theorem (cf. [En, 1.13.2], [Ko]), we may assume that $X$ is the inverse limit of the inverse sequence

$$
\left|K_{1}\right| \stackrel{f_{1}}{\longleftarrow}\left|K_{2}\right| \stackrel{f_{2}}{\longleftarrow}\left|K_{3}\right| \stackrel{f_{3}}{\longleftarrow} \cdots
$$

such that mesh $f_{i, \infty}^{-1}\left(K_{i}\right) \rightarrow 0(i \rightarrow \infty)$, where each $f_{i, \infty}: X \rightarrow\left|K_{i}\right|$ is the projection, each $K_{i}$ is a finite simplicial complex with $\operatorname{dim} K_{i} \leqslant \operatorname{dim} X \leqslant n$ and each $f_{i}$ is PL (piece-wise linear).

Let $K_{0}=\left\{v_{0}\right\}$ be the simplicial complex consisting of only one vertex. Then the constant map $f_{0}: K_{1} \rightarrow K_{0}$ is simplicial. Let $K_{1}^{\prime}=K_{1}$ and inductively choose simplicial subdivisions $K_{i}^{\prime}$ and $K_{i}^{*}$ of $K_{i}$ so that $K_{i}^{*}$ is a subdivision of the barycentric subdivision of $K_{i}^{\prime}$ and $f_{i}: K_{i+1}^{\prime} \rightarrow K_{i}^{*}$ is simplicial.

Let $M_{1}$ be the $n$-skeleton of

$$
M\left(f_{0}\right) \times L_{1} \cup_{K_{1} \times L_{1}} K_{1} \times\left(v_{1} * L_{1}\right),
$$

which is $(n-1)$-connected. We regard $K_{1}^{*}$ as a subdivision of $K_{1} \times\left\{v_{1}\right\}$. Then $M_{1}$ has the simplicial subdivision $M_{1}^{*}$ with $\left(M_{1}^{*}\right)^{(0)}=\left(M_{1}\right)^{(0)} \cup\left(K_{1}^{*}\right)^{(0)}$ which contains $M\left(f_{0}\right) \times L_{1}$ and $K_{1}^{*}$ as subcomplexes. Using the $G_{1}$-actions on $L_{1}$ and $v_{1} * L_{1}$, we define a simplicial $G_{1}$-action on $M_{1}^{*}$ by $g(x, y)=(x, g y)$ on $\left|M\left(f_{0}\right)\right| \times\left|L_{1}\right|$ and $\left|K_{1}\right| \times\left|v_{1} * L_{1}\right|$. Observe that $\left|K_{1}\right|=\left|K_{1}\right| \times\left\{v_{1}\right\}$ is the fixed point set of every $g \in G_{1} \backslash\left\{e_{1}\right\}$. Let $N_{1,1}=M_{1}^{*}$ and define $N_{1, i+1}$ inductively as the $n$-skeleton of $N_{1, i} \times L_{i+1}$ and let $p_{1, i}:\left|N_{1, i+1}\right| \rightarrow\left|N_{1, i}\right|$ be the projection. Then we have the inverse sequence

$$
\left|N_{1,1}\right| \stackrel{p_{1,1}}{\longleftarrow}\left|N_{1,2}\right| \stackrel{p_{1,2}}{\longleftarrow}\left|N_{1,3}\right| \stackrel{p_{1,3}}{\longleftarrow} \cdots
$$

such that the inverse limit $N_{1}$ is a compact $\mu^{n}$-manifold as is shown in [Sa] by using [GHW, Theorem 1]. Since $\left|N_{1,1}\right|=\left|M_{1}\right|$ is $(n-1)$-connected, $N_{1}$ is indeed homeomorphic to $\mu^{n}$.

Note that $f_{1}: K_{2}^{\prime} \rightarrow K_{1}^{*}$ is simplicial. Let $i_{1}: K_{1}^{*} \subset M_{1}^{*}=N_{1,1}$ be the inclusion. Then $i_{1} f_{1}: K_{2}^{\prime} \rightarrow N_{1,1}$ is also simplicial and $M\left(i_{1} f_{1}\right)=N_{1,1} \cup_{K_{1}^{*}} M\left(f_{1}\right)$. The $G_{1}$-action on $N_{1,1}$ extends simplicially to $M\left(i_{1} f_{1}\right)$ so that $\left|M\left(f_{1}\right)\right|$ is the fixed point set for every $g \in G_{1} \backslash\left\{e_{1}\right\}$.

Let $M_{2}$ be the $n$-skeleton of

$$
M\left(i_{1} f_{1}\right) \times L_{2} \cup_{K_{2}^{\prime} \times L_{2}} K_{2}^{\prime} \times\left(v_{2} * L_{2}\right),
$$

which is $(n-1)$-connected. We regard $K_{2}^{*}$ as a subdivision of $K_{2}^{\prime} \times\left\{v_{2}\right\} \subset M_{2}$. Then $M_{2}$ has the simplicial subdivision $M_{2}^{*}$ with $\left(M_{2}^{*}\right)^{(0)}=\left(M_{2}\right)^{(0)} \cup\left(K_{2}^{*}\right)^{(0)}$ which contains $M\left(i_{1} f_{1}\right) \times L_{2}$ and $K_{2}^{*}$ as subcomplexes. Observe that $N_{1,2}=M_{1}^{*} \times L_{2} \subset$ 
$M\left(i_{1} f_{1}\right) \times L_{2} \subset M_{2}^{*}$. Using the $G_{1}$-action on $M\left(i_{1} f_{1}\right)$ and the $G_{2}$-actions on $L_{2}$ and $v_{2} * L_{2}$, we define a simplicial semi-free action of $G_{2}^{\prime}$ on $M_{2}^{*}$ by $\left(g_{1}, g_{2}\right)\left(x_{1}, x_{2}\right)=$ $\left(g_{1} x_{1}, g_{2} x_{2}\right)$ on $\left|M\left(i_{1} f_{1}\right)\right| \times\left|L_{2}\right|$ and $\left|K_{2}\right| \times\left|v_{2} * L_{2}\right|$. Then $\left|K_{2}\right|=\left|K_{2}\right| \times\left\{v_{2}\right\}$ is the fixed point set of every $\left(g_{1}, g_{2}\right) \in G_{2}^{\prime} \backslash\left\{e_{2}^{\prime}\right\}$. In other words, this action induces the free $G_{2}^{\prime}$-action on $\left|M_{2}\right| \backslash\left|K_{2}\right|$. We have a retraction $r_{1}=c_{1} p_{1}:\left|M_{2}\right| \rightarrow\left|M_{1}\right|$, where $p_{1}:\left|M_{2}\right| \rightarrow\left|M\left(i_{1} f_{1}\right)\right|$ is the projection and $c_{1}:\left|M\left(i_{1} f_{1}\right)\right| \rightarrow\left|M_{1}\right|$ is the collapsing. Then $r_{1}\left(\left(g_{1}, g_{2}\right) x\right)=g_{1} r_{1}(x)$ for each $x \in\left|M_{2}\right|$ and $\left(g_{1}, g_{2}\right) \in G_{2}^{\prime}, r_{1}$ induces an isomorphism of homotopy groups of dimension $\leqslant n-1$ and $r_{1}|| K_{2} \mid=f_{1}$. Let $N_{2,1}=M_{2}^{*}$ and define $N_{2, i+1}$ inductively as the $n$-skeleton of $N_{2, i} \times L_{i+2}$ and let $p_{2, i}:\left|N_{2, i+1}\right| \rightarrow\left|N_{2, i}\right|$ be the projection. Then $N_{1, i+1} \subset N_{2, i}$ for each $i \in \mathbb{N}$. Similarly as above, we have the inverse sequence

$$
\left|N_{2,1}\right| \stackrel{p_{2,1}}{\longleftarrow}\left|N_{2,2}\right| \stackrel{p_{2,2}}{\longleftarrow}\left|N_{2,3}\right| \stackrel{p_{2,3}}{\longleftarrow} \cdots
$$

such that the inverse limit $N_{2}$ is homeomorphic to $\mu^{n}$.

Note that $f_{2}: K_{3}^{\prime} \rightarrow K_{2}^{*}$ is simplicial. Let $i_{2}: K_{2}^{*} \subset M_{2}^{*}=N_{2,1}$ be the inclusion. Then $i_{2} f_{2}: K_{3}^{\prime} \rightarrow N_{2,1}$ is also simplicial and $M\left(i_{2} f_{2}\right)=N_{2,1} \cup_{K_{2}^{*}} M\left(f_{2}\right)$. The $G_{2}^{\prime}$-action on $N_{2,1}$ extends simplicially to $M\left(i_{2} f_{2}\right)$ so that $\left|M\left(f_{2}\right)\right|$ is the fixed point set for every $g \in G_{2}^{\prime} \backslash\left\{e_{2}^{\prime}\right\}$.

By induction, we have the following diagram of the inverse sequences:

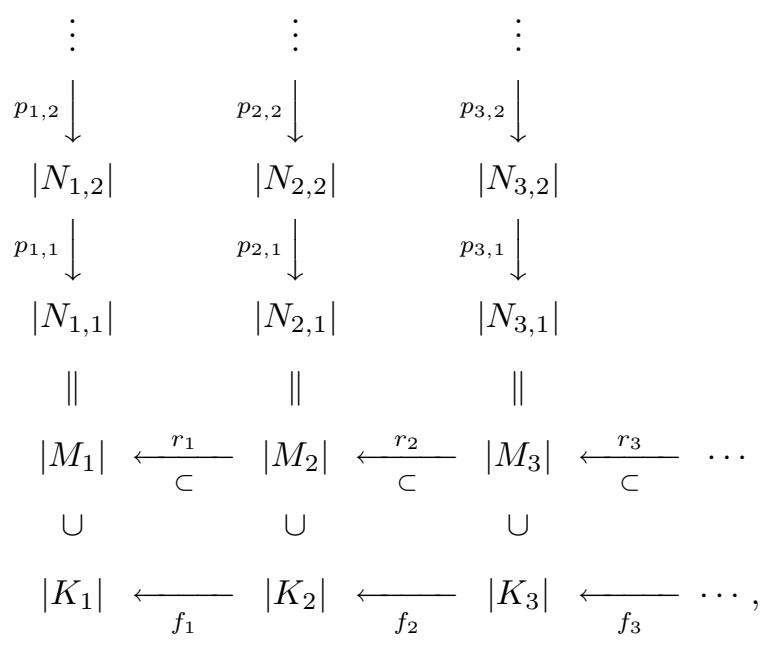

where the following conditions are satisfied:

(1) each $\left|M_{i}\right|$ is $(n-1)$-connected,

(2) each $N_{i, 1}$ is a subdivision of $M_{i}$ which has a simplicial $G_{i}^{\prime}$-action,

(3) each $\left|K_{i}\right|$ is the fixed point set of every $g \in G_{i}^{\prime} \backslash\left\{e_{i}^{\prime}\right\}$,

(4) $r_{i}$ is a retraction such that $r_{i}|| K_{i+1} \mid=f_{i}$ and

$$
r_{i}\left(\left(g_{1}, \cdots, g_{i+1}\right) x\right)=\left(g_{1}, \cdots, g_{i}\right) r_{i}(x)
$$

for each $x \in\left|M_{i}\right|$ and $\left(g_{1}, \cdots, g_{i+1}\right) \in G_{i}^{\prime}$,

(5) $r_{i}$ induces an isomorphism of homotopy groups of dimension $\leqslant n-1$,

(6) $N_{i, j} \subset N_{i+1, j-1} \subset \cdots \subset N_{i+j-1,1}, p_{i, j}=r_{i+j-1}|| N_{i, j+1} \mid$ and

(7) the inverse limit $N_{i}$ of the inverse sequence

$$
\left|N_{i, 1}\right| \stackrel{p_{i, 1}}{\longleftarrow}\left|N_{i, 2}\right| \stackrel{p_{i, 2}}{\longleftarrow}\left|N_{i, 3}\right| \stackrel{p_{i, 3}}{\longleftarrow} \cdots
$$

is homeomorphic to $\mu^{n}$. 
Let $M$ be the inverse limit of the sequence

$$
\left|M_{1}\right| \stackrel{r_{1}}{\longleftarrow}\left|M_{2}\right| \stackrel{r_{2}}{\longleftarrow}\left|M_{3}\right| \stackrel{r_{3}}{\longleftarrow} \cdots .
$$

We can regard $X$ and each $N_{i}$ as subspaces of $M$. Observe that

$$
\begin{gathered}
M \backslash X=\bigcup_{i \in \mathbb{N}} N_{i}=\bigcup_{i \in \mathbb{N}} \operatorname{int}_{M} N_{i} \text { and } \\
\operatorname{int}_{M} N_{i}=r_{i \infty}^{-1}\left(\left|M_{i}\right| \backslash\left|K_{i}\right|\right),
\end{gathered}
$$

where $r_{i \infty}: M \rightarrow\left|M_{i}\right|$ is the projection. Hence $M \backslash X$ is a $(n-1)$-connected $\mu^{n}$-manifold. It is easy to see that $M$ is $L C^{n-1}$ and $X$ is a $Z$-set in $M$. Then $M$ is $(n-1)$-connected. By Bestvina's characterization of $\mu^{n}$ [Be, 5.2.3], $M$ is homeomorphic to $\mu^{n}$.

We define an action of $G \subset \prod_{i \in \mathbb{N}} G_{i}$ on $M \subset \prod_{i \in \mathbb{N}}\left|M_{i}\right|$ as follows:

$$
\left(g_{1}, g_{2}, \cdots\right)\left(x_{1}, x_{2}, \cdots\right)=\left(g_{1} x_{1},\left(g_{1}, g_{2}\right) x_{2},\left(g_{1}, g_{2}, g_{3}\right) x_{3}, \cdots\right) \text {. }
$$

Each $x=\left(x_{1}, x_{2}, \cdots\right) \in X$ is a fixed point of every $g=\left(g_{1}, g_{2}, \cdots\right) \in G$ since $x_{i} \in\left|K_{i}\right|$ is a fixed point of $\left(g_{1}, \cdots, g_{i}\right) \in G_{i}^{\prime}$ for each $i \in \mathbb{N}$. On the other hand, $g x \neq x$ for each $x=\left(x_{1}, x_{2}, \cdots\right) \in M \backslash X$ and $g=\left(g_{1}, g_{2}, \cdots\right) \in G \backslash\{e\}$. In fact, $x \in \operatorname{int}_{M} N_{i}$ for some $i \in \mathbb{N}$. Let

$$
\begin{gathered}
x_{i+1}=\left(x_{i}, x_{i+1}^{\prime}\right) \in\left|M_{i}\right| \times\left|L_{i+1}\right|, \\
x_{i+2}=\left(x_{i}, x_{i+1}^{\prime}, x_{i+2}^{\prime}\right) \in\left|M_{i}\right| \times\left|L_{i+1}\right| \times\left|L_{i+2}\right|,
\end{gathered}
$$

Identifying $x=\left(x_{i}, x_{i+1}^{\prime}, x_{i+2}^{\prime}, \cdots\right) \in\left(\left|M_{i}\right| \backslash\left|K_{i}\right|\right) \times \prod_{j>i}\left|L_{j}\right|$,

$$
\begin{aligned}
g x & =\left(\left(g_{1}, \cdots, g_{i}\right) x_{i}, g_{i+1} x_{i+1}^{\prime}, g_{i+2} x_{i+2}^{\prime}, \cdots\right) \\
& \neq\left(x_{i}, x_{i+1}^{\prime}, x_{i+2}^{\prime}, \cdots\right)=x
\end{aligned}
$$

because the $G_{i}^{\prime}$-action on $\left|M_{i}\right| \backslash\left|K_{i}\right|$ and the $G_{j}$-action on $\left|L_{j}\right|(j>i)$ are free. Therefore $X$ is the fixed point set for every $g \in G \backslash\{e\}$. Since $X$ is a $Z$-set in $M$, we have the result by the $Z$-set unknotting theorem [Be, 3.1.5].

Concerning our result, the following question arises:

Question. If $X$ is a closed set in $\mu^{n}$ but not a $Z$-set, is the theorem still true?

Remark. This question has been solved affirmatively by Iwamoto. In his paper "Fixed point sets of transformation groups of Menger manifolds, their pseudointerior and their pseudo-boundaries" [Topology Appl. 68 (1996), 267-283], by extending the method of this paper, he proved that if $M$ is a $\mu^{n}$-manifold and $X$ is a closed set in $M$ then there exists a semi-free $G$-action on $M$ such that $X$ is the fixed point set of every $g \in G \backslash\{e\}$. Moreover it is also proved that $M$ has a $G$-invariant pseudo-interior $\nu(M)$. Then we have the same result for any pseudo-interior $\nu(M)$ of a $\mu^{n}$-manifold $M$.

\section{REFERENCES}

[Be] M. Bestvina, Characterizing k-dimensional universal Menger compacta, Memoirs Amer. Math. Soc. (no.380) 71 (1988). MR 89g:54083

[En] R. Engelking, Dimension Theory, N.-H. Math. Library vol. 19, North-Holland Publ. Co., Amsterdam, 1978. MR 58:2753b 
[Dr] A.N. Dranishnikov, On free actions of zero-dimensional compact groups, Izv. Akad. Nauk SSSR, Ser. Mat. 32 (1989), 217-232 (Russian), English transl. in: Math. USSR Izvestiya. MR 90e:57065

[GHW] D.J. Garity, J.P. Henderson and D.G. Wright, Menger spaces and inverse limits, Pacific J. Math. 131 (1988), 249-259. MR 89d:54026

[Ko] Y. Kodama, On embeddings of spaces into ANR and shape, J. Math. Soc. Japan 27 (1975), 533-544. MR 53:3993

[Po] L.S. Pontryagin, Topological Groups, Gordon and Breach, New York, 1966. MR 34:1439

[Sa] K. Sakai, Free actions of zero-dimensional compact groups on Menger manifolds, Proc. Amer. Math. Soc. 122 (1994), 647-648. MR 95c:57057

[Wh] J.H.C. Whitehead, Simplicial spaces, nuclei, and m-groups, Proc. London Math. Soc. (2) 45 (1939), 243-327.

Institute of Mathematics, University of Tsukuba, Tsukuba-city 305, Japan

E-mail address: sakaiktr@sakura.cc.tsukuba.ac.jp 